\title{
La biologie dans la formation des enseignants du premier degré : quelle part de spécificité?
}

\section{Patricia Schneeberger}

\section{(2) OpenEdition \\ 1 Journals}

\section{Édition électronique}

URL : http://journals.openedition.org/trema/2019

DOI : $10.4000 /$ trema.2019

ISSN : 2107-0997

\section{Éditeur}

Faculté d'Éducation de l'université de Montpellier

\section{Édition imprimée}

Date de publication : 1 septembre 1996

Pagination : 65-71

ISSN : 1167-315X

\section{Référence électronique}

Patricia Schneeberger, « La biologie dans la formation des enseignants du premier degré : quelle part de spécificité ? », Tréma [En ligne], 9-10 | 1996, mis en ligne le 11 septembre 2013, consulté le 10 décembre 2020. URL : http://journals.openedition.org/trema/2019; DOI : https://doi.org/10.4000/ trema.2019

Ce document a été généré automatiquement le 10 décembre 2020.

Trema 


\title{
La biologie dans la formation des enseignants du premier degré : quelle part de spécificité ?
}

\author{
Patricia Schneeberger
}

\section{Introduction}

1 En une vingtaine d'années (de 1969 à 1995), les textes qui définissent l'enseignement des sciences en général, et de la biologie en particulier, à l'école élémentaire en France ont changé plusieurs fois. On est ainsi passé des leçons de choses aux activités d'éveil (1969) pour revenir en 1985 à une vision plus traditionnelle. En 1995, de nouveaux programmes sont proposés où apparaît l'idée d'une émergence progressive des différents domaines disciplinaires. C'est ainsi qu'au cycle 2, on parle de « découverte du monde » (où figure une partie intitulée "le monde du vivant » à côté d'autres domaines comme «le monde de la matière et des objets » ou « le temps dans la vie des hommes »), alors qu'au cycle 3 s'effectue la différenciation des disciplines. Du point de vue des enseignants de l'école primaire, cela revient à permettre aux enfants de passer d'une approche globale à une approche disciplinaire. Sans être tout à fait nouvelle, cette mesure oblige à repenser la formation professionnelle en y intégrant une réflexion sur la spécificité des différentes disciplines enseignées et sur les convergences des approches qu'elles proposent.

\section{Une situation paradoxale}

2 Si la plupart des futurs professeurs des écoles ne sont pas des spécialistes de la biologie, ils disposent déjà, du fait de leur mode de recrutement, de cadres de pensée liés aux différentes disciplines (dont la biologie) construites au cours de leur passé scolaire. Le contexte de la formation les place face à un formateur spécialiste d'une discipline dans le cadre d'un module spécialisé, ils ont donc tendance à mobiliser la grille de lecture 
correspondant au domaine considéré, sans même s'apercevoir qu'un même concept (l'énergie par exemple) a une signification différente selon la discipline qui l'utilise.

Il convient donc de préciser la nature du questionnement qui fait la spécificité de la biologie afin d'inscrire les savoirs propres à cette discipline non comme un donné mais comme un construit. À partir de là, on peut espérer mieux concilier approche disciplinaire et formation si les formés sont amenés à comprendre ce qui fonde cette discipline (en termes d'objets, de tâches, des savoirs, de matrice pour reprendre la définition de M. Develay). À cela s'ajoute la nécessité de clarifier les valeurs qui président à l'organisation de l'enseignement de la biologie afin d'éclairer la pratique des enseignants.

4 Par ailleurs, il ne faut pas perdre de vue que nous formons des généralistes et la polyvalence des maitres conduit à considérer le problème de la complémentarité des modules disciplinaires proposés en formation.

5 Il convient donc de s'intéresser à l'idée que se font les futurs enseignants du premier degré de la spécificité de la biologie avec pour objectif de les aider à porter un regard distancié sur cette discipline prise comme objet d'enseignement.

\section{Conceptions des futurs enseignants}

\subsection{Influence de la formation antérieure}

6 Tous les futurs enseignants que nous formons à l'IUFM ont reçu au cours de leur scolarité un enseignement de la biologie inscrite comme discipline obligatoire, au collège puis éventuellement optionnelle au lycée. Cette formation antérieure a laissé des traces, bien que certains prétendent ne plus avoir beaucoup de souvenirs du contenu de cet enseignement.

7 Quand on interroge les professeurs des écoles stagiaires sur leurs souvenirs de l'enseignement qui leur a été dispensé en biologie, ils citent, le plus souvent en premier, l'étude des grandes fonctions (respiration, reproduction, ...) qu'ils considèrent comme caractéristiques de tout être vivant. Ils évoquent également, et cela s'accentue ces dernières années, les relations entre les êtres vivants et leur milieu.

Peut-on établir un lien entre ce que les élèves ont retenu en priorité et la façon dont la biologie leur a été présentée au cours de leur scolarité ? On peut tout au moins supposer l'influence d'un enseignement longtemps basé sur l'organisation des êtres vivants et visant à expliquer comment sont réalisées les fonctions caractéristiques du vivant. On a plus récemment assisté à ce que $\mathrm{M}$. Develay appelle un changement de matrice disciplinaire dans l'enseignement secondaire où l'on est passé à une vision plus systémique prenant davantage en compte les différentes formes d'échanges d'un organisme avec son milieu.

9 L'idée de principe organisateur de l'enseignement de la biologie nous intéresse d'autant qu'il paraît important de rechercher avec les futurs enseignants ce qui fait l'identité d'une discipline scolaire. Nous pensons avec M. Develay que les professeurs doivent aborder une telle réflexion épistémologique "afin de mettre en cohérence objets d'enseignement, méthodes et techniques d'enseignement, théories de référence ".

Par ailleurs, les matrices diffèrent selon le niveau d'enseignement et si l'on veut, comme le soulignent les nouveaux programmes de l'école primaire, assurer la continuité des 
apprentissages, il est nécessaire que les enseignants sachent identifier ce qui structure chacune des disciplines qu'ils enseignent. Il en résulte que les formateurs doivent les aider à analyser les programmes de chaque niveau d'enseignement afin de dégager les cadres de référence correspondants. Ils pourront ainsi mieux situer le point de vue qu'ils doivent adopter pour enseigner une discipline à un niveau donné, éventuellement prendre conscience de l'influence de l'enseignement qu'ils ont reçu et envisager les changements qu'ils devront eux-mêmes opérer dans l'idée qu'ils se font d'une discipline scolaire. En précisant ainsi les différentes matrices disciplinaires, on devrait permettre aux enseignants de mieux préparer leurs élèves à leur scolarité ultérieure.

\subsection{Premiers résultats d'une enquête}

11 Dans le but d'aider les futurs professeurs des écoles à caractériser la biologie qu'ils vont enseigner, nous avons demandé à plusieurs groupes d'étudiants et de stagiaires en formation initiale de rechercher les aspects spécifiques de cette discipline et de dégager quelques points communs avec les autres disciplines scientifiques (physique et technologie). Pour cela, nous leur avons proposé un questionnaire (portant sur les points évoqués ci-dessus) à remplir individuellement puis nous avons organisé des travaux de groupes, afin qu'ils comparent leurs points de vue respectifs. Le dépouillement des réponses au questionnaire ainsi que le bilan des échanges entre les formés donne un aperçu de la façon dont les futurs enseignants situent la Biologie et son enseignement par rapport à d'autres disciplines.

12 Une analyse des réponses obtenues auprès de 82 formés a permis de dégager certaines tendances, même si des différences existent entre les personnes interrogées, et notamment selon que l'on s'adresse aux étudiants de première année d'IUFM ou aux professeurs des écoles stagiaires (deuxième année d'IUFM) qui n'ont pas le même profil du fait de leur cursus antérieur. Toutefois, l'enquête ainsi réalisée mériterait d'être poursuivie et affinée afin de préciser ces premiers résultats.

13 La plupart de nos formés semblent considérer la biologie comme une science qui étudie le monde vivant c'est-à-dire la faune, la flore et l'Homme et, pour certains, les relations que réalisent les êtres vivants entre eux et avec leur milieu. Selon cette acception, la biologie s'intéresserait aux êtres vivants plutôt qu'au concept de vie, même s'ils précisent qu'il s'agit de comprendre comment fonctionnent les organismes vivants (à travers l'étude des grandes fonctions). Ainsi, par exemple, à l'issue d'un échange, certains stagiaires ont comparé la biologie à la physique : ils ont défini la biologie comme une approche concrète des phénomènes naturels, considérant la physique comme une approche plus théorique basée sur la recherche de lois, de principes et sur une «modélisation plus poussée ». Ils différencient donc ces deux disciplines selon le degré d'abstraction qu'elles supposent, d'après eux. Nous avons retrouvé cette idée dans les réponses données au questionnaire par 11 des 42 stagiaires (deuxième année d'IUFM) interrogés.

14 Notons également que la génétique est très peu citée ( 5 personnes sur 82 ) parmi les domaines d'étude de la biologie. Si je précise ce point, c'est à cause de l'importance qu'occupe la notion de gène et d'information génétique dans les nouveaux programmes de lycée (cf. la communication de J.-C. Hervé).

Si les stagiaires caractérisent essentiellement la biologie par son objet d'étude, ils évoquent également les méthodes (et éventuellement quelques techniques) qu'elle utilise: ils citent souvent la démarche expérimentale basée pour la plupart sur 
l'expérimentation (l'observation est moins souvent citée) et, dans une proportion plus faible, le raisonnement qualifié de scientifique.

L'idée que se font les futurs enseignants de l'école élémentaire, de la biologie, est un facteur qui risque vraisemblablement de déterminer la façon dont ils vont organiser leur enseignement. Quand on leur demande ce qui leur paraît important d'enseigner à l'école élémentaire, leurs choix portent le plus souvent sur les domaines d'étude à envisager (faune, flore, corps humain, environnement). Pour eux, semble-t-il, les priorités de cet enseignement correspondraient à l'acquisition des connaissances (et aussi pour certains, des méthodes), essentielles, afin de comprendre comment fonctionne notre corps et de découvrir le monde qui nous entoure.

\section{Définir la Biologie}

Selon les programmes de l'école primaire, l'enseignant doit permettre aux élèves de découvrir le monde vivant; en abordant l'étude du corps de l'enfant et les principales manifestations de la vie animale et végétale, il peut dégager progressivement les caractéristiques du vivant. Cela suppose que l'enseignant connaisse au préalable ces caractéristiques et donc qu'il ait une vue d'ensemble sur cette discipline qu'est la biologie.

\subsection{Les demandes des enseignants}

18 Au cours de contacts que nous avons avec les enseignants de l'école primaire (stages de formation continue et collaborations diverses), le problème de la délimitation du champ d'investigation propre à la biologie est souvent abordé. L'évolution de cette discipline, tant du point de vue de la recherche que de l'enseignement, et les échos que les instituteurs en ont, notamment par les médias, les désorientent. Ils nous demandent alors de leur préciser quels sont les critères qui permettent d'affirmer si l'on se place dans le champ de la biologie quand on propose aux élèves tel ou tel type d'activité. En maternelle, par exemple, le découpage des disciplines n'ayant pas lieu d'être, les enseignants ont parfois des difficultés à situer le contenu de leur enseignement. Souvent, même lorsqu'ils enseignent au cycle 3 où les disciplines sont différenciées, les enseignants distinguent mal les spécificités de chaque discipline (en termes de concepts, de valeurs, de techniques) alors que leur enseignement peut comporter des éléments communs (en terme d'objectifs ou de démarche pédagogique).

Une aide des formateurs est donc nécessaire ; reste à trouver quels sont les moyens qui permettront d'éclairer les pratiques des enseignants.

\subsection{Quelle réponse apporter aux enseignants ?}

Dire que la biologie étudie les êtres vivants, leur organisation et leur comportement ne suffit pas à comprendre ce qui fonde cette discipline. En effet, cette définition ne permet plus actuellement de reconnaître la permanence de la biologie à travers les programmes des différents niveaux d'enseignement. Par ailleurs, les termes unité, diversité ou complexité généralement utilisés pour caractériser le monde vivant ne peuvent plus être retenus pour définir la spécificité de la biologie car ces termes s'appliquent également aux objets d'étude de la physique ou de la technologie. 
21 Le fait de discourir sur un être vivant (un animal familier par exemple), comme c'est le cas lors de certaines séquences de langage à l'école maternelle, ne peut être considéré comme une activité relevant de la biologie car l'enseignement de la biologie ne peut, en effet, se réduire à l'introduction d'un vocabulaire spécifique.

Certes les formateurs peuvent aider les enseignants à surmonter certaines des difficultés rencontrées lors de la préparation des séquences d'enseignement: définitions des objectifs, prise en compte des problèmes d'ordre pratique qui nécessitent parfois l'apprentissage de techniques spécifiques (élevages ou cultures par exemple). Cependant, les formateurs ont eux-mêmes des difficultés pour définir la spécificité de la discipline qu'ils sont censés représenter, même s'il leur est facile d'identifier, par simple habitude, ce qui appartient à la biologie et ce qui relève d'une autre discipline.

S'ils savent reconnaître les problèmes auxquels la biologie peut apporter des explications, les spécialistes de cette discipline ont souvent tendance à privilégier les réponses oubliant parfois de préciser les questions correspondantes. Or, selon M. Develay « une discipline n'existe qu'en réponse à des questions données, qui proviennent de la nature du regard que nous portons sur le monde qui nous entoure ». Si nous adoptons ce point de vue, il s'agit alors de préciser la nature du questionnement que l'on utilise quand on fait intervenir la biologie pour expliquer le monde qui nous entoure. C'est à ce prix que nous pourrons aider les enseignants à concevoir un enseignement qui permette aux élèves de construire progressivement l'approche disciplinaire propre à la biologie.

\section{Un projet de formation}

24 À l'IUFM d'Aquitaine, des modules dits « de réajustement » sont proposés aux professeurs des écoles stagiaires (deuxième année de formation). Il s'agit de compléments de formation dans des domaines disciplinaires choisis en option au cours de la première année (axée sur la préparation au concours). Les enseignants de Biologie-Géologie, de Physique et de Technologie des différentes antennes départementales de l'IUFM se sont associés pour élaborer un module pluridisciplinaire. Les objectifs retenus pour cette formation sont essentiellement d'ordre professionnel puisqu'il s'agit principalement de permettre aux élèves-professeurs de «mettre en pratique la polyvalence dans le cadre des activités scientifiques et technologiques». Pour cela, on demande aux formés de construire et de mettre en place dans des classes un projet qui prenne en compte les convergences et les spécificités de chaque discipline au sein du domaine des Sciences et Technologie.

Les sujets d'étude envisagés doivent naturellement permettre, d'une part, de faire apparaître des points communs entre les différents secteurs constituant les sciences et technologie et, d'autre part, de mettre en œuvre des approches spécifiquement disciplinaires. C'est la raison pour laquelle l'équipe de formateurs a proposé de s'intéresser aux thèmes relatifs à l'éducation pour l'environnement qui nécessite une approche globale faisant appel à plusieurs disciplines.

Par ailleurs, en abordant les grands problèmes du monde contemporain (la pollution, les nuisances, la gestion de l'eau, ...), nous espérons également, chemin faisant, développer la curiosité des futurs professeurs des écoles et renouveler leur intérêt pour les domaines des sciences et des techniques. 
Ce module a été mis en place à la rentrée 1995 et a reçu un accueil favorable de la part des élèves-professeurs. Nous considérons, quant à nous, que ce projet, actuellement en phase d'expérimentation, devrait aider les futurs enseignants à mieux caractériser les différentes approches disciplinaires. L'évaluation des effets de cette formation nous indiquera si nous avons réussi à atteindre cet objectif.

\section{Conclusion}

En proposant ma participation à une table ronde sur la spécificité de la biologie ou plus exactement sur la spécificité de l'enseignement de la biologie à l'école primaire, je pensais d'avantage entamer une réflexion sur des questions qui m'apparaissent cruciales pour la formation des maitres. Ma contribution n'avait pas pour but de proposer des solutions car mes collègues de l'IUFM et moi-même avons encore peu avancé dans ce domaine.

Si les enseignants de l'école primaire doivent réellement préparer les élèves au collège, ce qui paraît aller de soi, ils doivent alors permettre à ceux-ci d'identifier les disciplines enseignées et pour cela il leur faut se familiariser avec les matrices correspondantes. Ils pourront peut-être ainsi réduire les conséquences d'un cloisonnement encore trop important des différents niveaux d'enseignement qui pose problème pour la biologie (comme pour d'autres disciplines certainement).

30 L'enjeu n'est pas dérisoire : il y va de l'efficacité de l'enseignement scientifique et donc de l'avenir des élèves dans un monde où les sciences sont omniprésentes.

La lutte contre l'alcoolisme, à l'école, sous la Troisième République. p. 130

DUVEAU G., Les Instituteurs, Collection : "Le temps qui court », 192 p., Paris, Éditions du Seuil, 1957, - Impr. par : Imprimerie Tardy-Quercy, Bourges.

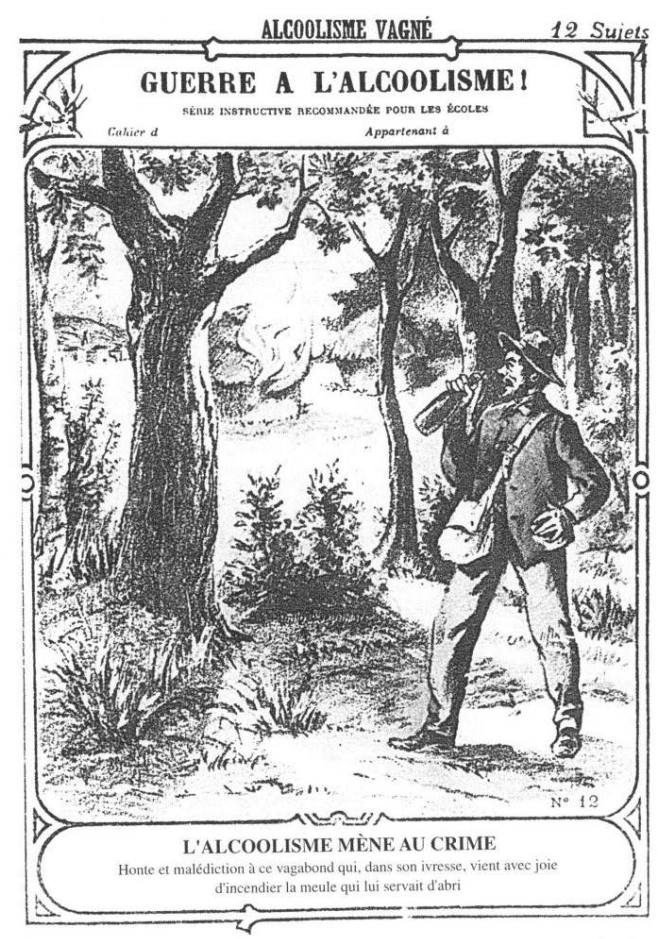




\section{BIBLIOGRAPHIE}

DEVELAY M., De l'apprentissage à enseignement, 165 p., Paris, E.S.F., 1992.

GIORDAN A. \& SOUCHON C., Une éducation pour l'environnement, 232 p, Nice, Z'éditions, 1992.

MINISTÈRE DE L'ÉDUCATION NATIONALE, Direction des Écoles, Programmes de l'école primaire, Paris, C.N.D.P., 1995.

\section{RÉSUMÉS}

La mise en place de trois cycles dans la scolarité primaire s'est accompagnée de nouveaux textes ministériels qui précisent la place de chacune des disciplines aux différents niveaux de l'enseignement. La biologie est incluse dans un ensemble de disciplines regroupées sous le terme «Découverte du monde Une réflexion s'impose donc sur la spécificité de la biologie et sur les apprentissages que suppose la différenciation des multiples approches disciplinaires.

The implementation of three stages within grade school instruction has gone band in band with new Ministry of Education legal texts specifying each discipline's place at the various levels of the teaching process. At grade school level, biology is included within an "assemblage" of disciplines grouped under the heading "Discovery of the world". It therefore becomes imperative, to reflect upon, the specific nature of biology and the learning processes implied by the differentiation of the various disciplinary approaches.

INDEX

Mots-clés : continuité des apprentissages, formation des maîtres

Keywords : continuity of learning process, teacher training

\section{AUTEUR}

PATRICIA SCHNEEBERGER

IUFM d'Aquitaine, LADIST, Université de Bordeaux I 